\title{
BMJ Open Sexually transmitted infections among high-risk populations that use treatment as prevention or pre-exposure prophylaxis: a protocol for a systematic review
}

\author{
Makella Coudray (D , , ${ }^{1}$ Sandra Kiplagat, ${ }^{1}$ Franklin Saumell, ${ }^{1}$ \\ Purnima Madhivanan ${ }^{2,3,4,5}$
}

To cite: Coudray M, Kiplagat S, Saumell F, et al. Sexually transmitted infections among high-risk populations that use treatment as prevention or pre-exposure prophylaxis: a protocol for a systematic review. BMJ Open 2019;9:e032054. doi:10.1136/ bmjopen-2019-032054

- Prepublication history for this paper is available online. To view these files, please visit the journal online (http://dx.doi. org/10.1136/bmjopen-2019032054).

$\mathrm{MC}$ and SK contributed equally.

MC and SK are joint first authors.

Received 01 June 2019 Revised 02 October 2019 Accepted 08 November 2019

Check for updates

(C) Author(s) (or their employer(s)) 2019. Re-use permitted under CC BY-NC. No commercial re-use. See rights and permissions. Published by BMJ.

For numbered affiliations see end of article.

Correspondence to Miss Makella Coudray; mcoud001@fiu.edu

\section{ABSTRACT}

Background Among men who have sex with men, preexposure prophylaxis (PrEP) reduces the risk of HIV by $95 \%$. Based on the documented benefits, the Centre for Disease Control and Prevention has recommended PrEP as a prevention method for high-risk groups. Moreover, for those HIV-infected individuals, antiretroviral therapy has been shown to serve as both as a treatment and prevention method for HIV.

Methods and analysis This systematic review protocol was reported according to the Preferred Reporting Items for Systematic reviews and Analyses (PRISMA) P framework. Medline (1980-present), Embase (1980-present), CINAHL (1980-present), Cochrane Central Register of Controlled Trials and clinicaltrials.gov will be used to identify relevant articles based on a piloted search strategy. Peer-reviewed observational and experimental studies will be included. A narrative style will be used to describe descriptive data. A meta-analysis will be conducted if heterogeneity is not significant.

Ethics and dissemination Recent evidence suggests that there is an increased risk of sexually transmitted infections (STIs) among high-risk persons that use PrEP. Furthermore, there is a paucity of data on the relationship of treatment as prevention and incidence of STIs. The findings of this review will assess this emerging public health phenomenon and serve to inform future public health policy. No formal ethical review is required for this protocol. All findings will be published in a peer reviewed journal.

PROTOCOL registration number CRD42019128720.

\section{INTRODUCTION}

According to the United Nations (UNAIDS), as of 2017 , there were 36.9 million people living with HIV globally. ${ }^{1}$ About 21.7 million of them were on antiretroviral therapy (ART). ${ }^{1}$ Advances in preventive measures for HIV have led to the use of ART by HIV-uninfected persons to prevent the acquisition of HIV, which is known as pre-exposure prophylaxis (PrEP). ${ }^{2}$ PrEP consists of a daily oral regimen
Strengths and limitations of this study

- This protocol was registered with PROSPERO, and Preferred Reporting Items for Systematic Reviews and Meta-Analyses (PRISMA) P guidelines were followed for reporting.

- Two reviewers will conduct screening and data extraction to reduce bias.

- Heterogeneity of results will be assessed.

Grey literature will be excluded.

of tenofovir alafenamide and emtricitabine..$^{3-5}$ PrEP has been shown to reduce the risk of acquisition of HIV. ${ }^{24}$ Among men who have sex with men, PrEP reduced the risk of HIV by $95 \% \cdot{ }^{36}$ The documented benefits of PrEP resulted in the Centre for Disease Control and Prevention (CDC) guidelines, which recommend PrEP as a prevention method for persons at high-risk for HIV. ${ }^{4} \mathrm{PrEP}$ is recommended for all populations; however, efficacy is influenced by the differential adherence among groups. ${ }^{2}$

Among HIV-infected individuals, ART has been shown to serve as both a treatment and prevention method for HIV. ART effectively decreases the viral load among HIV-infected persons who are adherent to treatment to an undetectable level, which prevents the transmission of HIV, also known as treatment as prevention (TasP). ${ }^{7}$ This phenomenon has been coined $\mathrm{U}=\mathrm{U}$, that is, undetectable $=$ untransmittable. TasP has been shown to reduce the risk of mother-to-child transmission of HIV and HIV transmission between HIV discordant couples. ${ }^{78}$ The risk of HIV transmission can be considered negligible among HIV-infected individuals with an undetectable 
viral load adherent to ART who are not coinfected with a sexually transmitted infection (STI) ${ }^{7}$

Literature illustrates that both PrEP and TasP decrease the risk of acquisition and transmission of HIV ${ }^{6}{ }^{9}$ High incidence rates of STIs among persons receiving PrEP and TasP are also documented. ${ }^{6}$ Jenness and colleagues suggest that elevated STI incidence among PrEP users is due to behavioural risk compensation and that PrEP use reduces the use of other disease prevention strategies. ${ }^{6}$ Current CDC guidelines recommend biannual screening for STIs among PrEP users. ${ }^{6}$ The use of PrEP has no biological effect on the risk of acquiring a bacterial STI. ${ }^{6}$ Literature is also unclear with respect to the relationship between PrEP and viral, bacterial and protozoal STIs. Marcus et al determined that PrEP was not a protective factor for herpes simplex virus 2 (HSV-2) acquisition, while Celum et al determined PrEP provided moderate protection against HSV-2. ${ }^{10}{ }^{11}$ There is a need to explore the behavioural predictors of increased STI incidence among PrEP users. There is a paucity of data with respect to TasP and STI incidence and testing among HIVinfected individuals. Consequently, the current systematic review aims to comprehensively assess available literature to elucidate the relationship between PrEP and TasP and the incidence of STIs.

\section{OBJECTIVES}

The objectives of this systematic review are as follows:

- To examine the use of TasP among HIV-infected persons and its associated risk of acquisition of STIs.

- To examine the use of PrEP among high-risk persons for HIV and the associated risk of acquisition of STIs.

- To compare the difference of the associated risk of acquisition of STIs between TasP and PrEP users.

\section{METHODS AND ANALYSIS}

Preferred Reporting Items for Systematic Reviews and Meta-Analyses (PRISMA) P guidelines were used to report this protocol. ${ }^{12}$ This systematic review will be reported according to the PRISMA guidelines. ${ }^{13}$

\section{Protocol registration}

This systematic review protocol was registered in the International Prospective Register of Systematic Reviews (PROSPERO) (registration number: CRD42019128720 Available from: http://www.crd.york.ac.uk/PROSPERO/ display_record.php?ID=CRD42019128720).

\section{Study selection}

Types of studies

Both observational and experimental study designs will be included (cohort, case-control, cross-sectional, case series, case reports and clinical studies).

\section{Types of participants}

Persons 12 years and older who are at-risk of transmitting HIV or becoming infected with HIV will be included in the study. Adolescents will be included since the Food and Drug Administration (FDA) has approved the use of PrEP by adolescents. ${ }^{14}$

Types of interventions

- TasP

- PrEP

Types of outcome measures

- Laboratory confirmed or self-reported, incident or prevalent STIs (Chlamydia trachomatis, Neisseria gonorrhoea, HSV-2, syphilis, human papillomavirus, hepatitis $\mathrm{C}$ virus, Trichomonas vaginalis).

\section{Eligibility criteria}

- Inclusion criteria

- Research findings published in peer-reviewed journals.

- Literature published since 2005.

- Exclusion criteria

- Animal studies

- Children (11 years and under)

- Grey literature

- Dissertations/theses

- Conference abstracts

- Studies without reported estimates (risk ratio, OR, CIs, point prevalence)

- Unpublished studies

- Protocols

\section{Search strategy}

The search strategy will include key-terms and databasespecific terminology, for example, Medical Subject Headings $(\mathrm{MeSH})$. We will be using databases including: Medline (1980-present), Embase (1980-present), CINAHL (1980-present), Cochrane Central Register of Controlled Trials and clinicaltrials.gov. We will modify the search strings based on the selected databases. Only studies that occurred since 2005 will be included. The first study which indicated that TasP was effective to reduce the transmission of HIV was published in $2011 .^{9}$ PrEP was approved by the FDA in 2012. ${ }^{15}$ As such only articles published since 2005 will be included in the review to capture research most relevant to TasP and PrEP interventions. The final PRISMA diagram will be presented. The general search strategy includes: PrEP or TaSP, STIs and HIV. Abbreviations used are included in table 1 We have included the overall search strategy in box 1. Boolean operators AND/OR will be included in the search strategy for the key terms and the MeSH words. The detailed strategy for MEDLINE is included in box 2. No language restrictions will be applied. The search strategy will be pilot tested and finalised.

All three independent reviewers (SK, MC and FS) will meet to identify the screening and data extraction process and conduct the literature search of all databases. The results of each database specific search strategy will be downloaded from the respective databases. We will then import the combined search results into Covidence, a reference software for full screen review and 


\begin{tabular}{ll} 
Table 1 & List of abbreviations \\
\hline ART & Antiretroviral therapy \\
CDC & Center for Disease Control and Prevention \\
FDA & Food and Drug Administration \\
HIV & human immunodeficiency virus \\
HSV-2 & herpes simplex virus 2 \\
MeSH & Medical Subject Headings \\
MSM & men who have sex with men \\
PrEP & Pre-exposure prophylaxis \\
PRISMA & Preferred Reporting Items for Systematic \\
& Reviews and Meta-Analyses \\
PROSPERO & Prospective Register of Systematic Reviews \\
STI & Sexually transmitted infection \\
TAF & Tenofovir alafenamide \\
TasP & Treatment as prevention \\
UNAIDS & United Nations \\
\hline
\end{tabular}

citations screening. ${ }^{16}$ Duplicates will be checked for and removed. An automated check for duplicate titles and year of publication will be applied. The authors will manually check each possible duplicate before removal to decrease error. The updated library will then be saved. Using Covidence, two independent reviewers (from among SK, MC and FS) will screen the titles and abstracts based on the eligibility criteria identified to determine which studies should be included for full text screening. If any disagreement occurs, two authors will discuss and resolve any issues. If no consensus is reached, the third author will arbitrate. Next, eligible full text articles will be screened by two independent reviewers (from among SK, MC and FS) for inclusion in the data extraction process. If any disagreement occurs, two authors will discuss and resolve any issues. If

\section{Box 1 Search strategy}

\section{Search query}

\#1 "treatment as prevention" OR TasP OR "pre-exposure prophylaxis" OR PrEP OR Tenofovir OR "emtricitabine-tenofovir" OR truvada OR descovy OR Emtriva OR FTC OR viread OR "tenofovir disoproxil fumarate" OR TDF OR coviracil OR PMPA OR TAF OR "tenofovir alafenamide" \#2 "sexually transmitted infection" OR "sexually transmitted infections" OR STI OR STIs OR "sexually transmitted disease" OR "Sexually transmitted diseases" OR STD OR STDs OR "venereal disease" OR "venereal diseases" OR chlamydia OR syphilis OR "herpes simplex virus type 2" OR HSV2 OR gonorrheagonorrhoea OR trichomoniasis OR "trichomonas vaginalis"

\#3 "Human Immunodeficiency VirusHIV" OR HIV OR "human immunodeficiency virusHIV infection" OR "acquired immunedeficiency syndrome" OR AIDS

OR "acquired immuno-deficiency syndrome" OR "human immunedeficiency virus" OR "human immune-deficiency virus"

\#4 Search (\#1 AND \#2 AND \#3)

\section{Box 2 Medline search strategy}

\#1 "treatment as prevention" OR TasP OR "pre-exposure prophylaxis" OR PrEP OR Tenofovir OR "emtricitabine-tenofovir" OR truvada OR descovy OR Emtriva OR FTC OR viread OR "tenofovir disoproxil fumarate" OR TDF OR coviracil OR PMPA OR MESH.EXACT("Pre-Exposure Prophylaxis") OR TAF OR "tenofovir alafenamide"

\#2 "sexually transmitted infection" $O R$ "sexually transmitted infections" OR STI OR STIs OR "sexually transmitted disease" OR "Sexually transmitted diseases" OR STD OR STDs OR "venereal disease" OR "venereal diseases" OR chlamydia OR syphilis OR "herpes simplex virus type 2" OR HSV2 OR gonorrheagonorrhoea OR trichomoniasis OR "trichomonas vaginalis" OR MESH.EXACT("Sexually Transmitted Diseases") OR MESH.EXACT.EXPLODE(“Chlamydia”) OR MESH. EXACT("Syphilis") OR MESH.EXACT("Herpes Genitalis") OR MESH. EXACT(“GonorrheaGonorrhoea") OR MESH.EXACT("Trichomonas Infections") OR MESH.EXACT(“Trichomonas Vaginitis")

\#3 "Human Immunodeficiency VirusHIV" OR HIV OR "human immunodeficiency virusHIV infection" OR "acquired immunedeficiency syndrome" OR AIDS

$\mathrm{OR}$ "acquired immuno-deficiency syndrome" OR "human immunedeficiency virus" OR "human immune-deficiency virus" $O R$ MESH.EXACT("HIV") OR MESH.EXACT(“Acquired Immunodeficiency SyndromeAIDS")

\#4 Search (\#1 AND \#2 AND \#3)

no consensus is reached, the third author will arbitrate. Reasons for exclusion will be referenced for each article at each stage.

\section{Data extraction}

Two independent reviewers (from among SK, MC, FS) will independently extract data using a piloted data extraction table from Microsoft Excel. The table will include study details such as title of the study, study design, study setting (including country), publication year, sample size, the intervention details and the outcome details such as HIV and STI incidence or prevalence (with types of laboratory tests to confirm HIV and STI diagnosis). Furthermore, the type of sampling techniques implemented by the study (convenience, snowball, trial and so on), the context of PrEP provision (informal or formal), condom use (before and after PrEP or TasP initiation) and PrEP regimen (daily, on-demand, event-based dosing, the Ts and Ss) will be assessed. These factors will be assessed to adequately evaluate factors which may moderate the association between treatment intervention and the acquisition of STIs.

The two reviewers will meet to resolve and discuss any disagreements. However, if disagreements persist, the third reviewer will arbitrate. For any missing information, the reviewers will contact the corresponding authors to request any updates on the missing items. Authors will be contacted a maximum of three times via email and/ or phone. Email will be the first form of contact. If unsuccessful, three attempts will then be made to call the authors. 


\section{Data management}

Covidence will be used for title and abstract screening and full-text screening. After each round of screening, a backup database will be saved. Reasons for exclusion of articles at each stage will be documented in Covidence.

\section{Data synthesis}

All data will be stratified by intervention type. TasP and PrEP populations will not be mixed. Findings for TasP and PrEP populations will be presented independently. Descriptive data will be summarised using a narrative style. A meta-analysis will be conducted if heterogeneity is not a major concern. The $\mathrm{I}^{2}$ statistic will be used to assess heterogeneity where $25 \%, 50 \%$ and $75 \%$ will represent low, moderate and high heterogeneity, respectively. ${ }^{17}$ Additionally, the $\chi^{2}$ test for heterogeneity will also be used. $\mathrm{P}<0.1$ will indicate significant heterogeneity. If heterogeneity is found, $\mathrm{p}$ values less than or equal to 0.05 will be considered statistically significant. A Forest plot will be used to graphically assess heterogeneity. The RevMan software will be used to generate the Forest Plot. Measures of association reported by studies to be included in the meta-analysis will be summarised using the random effects model for meta-analysis. If heterogeneity is found, sensitivity and subgroup analyses will be performed. The $\mathrm{R}$ Project for Statistical Computing will be used to conduct analyses.

\section{Quality assessment}

The Quality Assessment Tool for Quantitative Studies will be used to assess the quality of articles to be included in the systematic review. Deeks et al determined this to be an effective assessment tool. ${ }^{18}$ Two reviewers (from among SK, MC and FS) will assess the quality of each of the included studies including items on selection bias, randomisation, participation, data collection and intervention integrity. If there are significant differences in scores, raters will discuss and resolve any differences. If differences cannot be resolved, a third rater will arbitrate. The quality of evidence will be assessed using the Grading of Recommendations Assessment, Development and Evaluation.

\section{Bias assessment}

Risk of bias will be assessed for each of the included studies using the Cochrane Collaboration's tool for the assessment of the risk of bias. ${ }^{19}$ RevMan will then be used to develop a funnel plot to graphically assess bias. Two independent reviewers (from among SK, MC and FS) will review each article with the tool including random sequence generation and allocation concealment (for selection bias), participant and personnel blinding (for performance bias), blinding of outcomes assessment (detection bias), incomplete data (attrition bias), selective reporting (reporting bias), as well as any other potential sources of bias. If there are disputes about a bias rating, raters will discuss and resolve any differences. If differences cannot be resolved a third rater will arbitrate.

\section{TIMELINE FOR SYSTEMATIC REVIEW}

Pilot screening was initiated from 14 March 2019. We anticipate that data extraction will begin December 2019. Our preliminary manuscript will be completed by July 2020 .

\section{Patient and public involvement}

No patient involved.

\section{ETHICS AND DISSEMINATION}

There are no formal ethics approvals needed for this review because we will only use data that is publicly available. The findings of this review will be published in a peer-reviewed journal. These findings will also be presented at relevant conferences. The PRISMA-P guidelines were used to report this protocol. Findings, as well as any amendments made, will also be reported using the PRISMA guidelines.

\section{Author affiliations}

${ }^{1}$ Epidemiology, Florida International University, Miami, Florida, USA

${ }^{2}$ Health Promotion Sciences, Mel \& Enid Zuckerman College of Public Health, University of Arizona, Tucson, Arizona, United States

${ }^{3}$ Internal Medicine, Division of Infectious Diseases, College of Medicine, University of Arizona, Tucson, Arizona, United States

${ }^{4}$ Family \& Community Medicine, College of Medicine, University of Arizona, Tucson, Arizona, United States

${ }^{5}$ Public Health Research Institute of India, Mysore, Karnataka, India

Acknowledgements The authors would like to acknowledge Barbara Sorondo for her assistance with developing a search strategy which was instrumental for the development of the protocol.

Contributors MC and SK: study concept and design, drafting of manuscript. FS: drafting of manuscript. PM: subject matter expertise, revision of manuscript, obtained funding

Funding MC and PM are supported by the National Institutes of Health grant (R15Al28714-01).

Competing interests None declared.

Patient consent for publication Not required.

Provenance and peer review Not commissioned; externally peer reviewed.

Data availability statement There are no data in this work.

Open access This is an open access article distributed in accordance with the Creative Commons Attribution Non Commercial (CC BY-NC 4.0) license, which permits others to distribute, remix, adapt, build upon this work non-commercially, and license their derivative works on different terms, provided the original work is properly cited, appropriate credit is given, any changes made indicated, and the use is non-commercial. See: http://creativecommons.org/licenses/by-nc/4.0/.

ORCID iD

Makella Coudray http://orcid.org/0000-0002-5906-3220

\section{REFERENCES}

1 UNAIDS. 2017 global HIV statistics, 2018. Available: http://www. unaids.org/en/resources/fact-sheet [Accessed 02 Apr 2019].

2 Desai M, Field N, Grant R, et al. Recent advances in pre-exposure prophylaxis for HIV. BMJ 2017;359. 
3 Chan PA, Glynn TR, Oldenburg CE, et al. Implementation of preexposure prophylaxis for human immunodeficiency virus prevention among men who have sex with men at a new England sexually transmitted diseases clinic. Sex Transm Dis 2016;43:717-23.

4 Tetteh RA, Yankey BA, Nartey ET, et al. Pre-Exposure prophylaxis for HIV prevention: safety concerns. Drug Saf 2017;40:273-83.

5 De Clercq E. Role of tenofovir alafenamide (TAF) in the treatment and prophylaxis of HIV and HBV infections. Biochem Pharmacol 2018;153:2-11.

6 Jenness SM, Weiss KM, Goodreau SM, et al. Incidence of gonorrhea and Chlamydia following human immunodeficiency virus preexposure prophylaxis among men who have sex with men: a modeling study. Clin Infect Dis 2017;65:712-8.

7 Dieffenbach CW, Fauci AS. Universal voluntary testing and treatment for prevention of HIV transmission. JAMA 2009;301:2380-2.

8 Anglemyer A, Rutherford GW, Horvath T, et al. Antiretroviral therapy for prevention of HIV transmission in HIV-discordant couples. Cochrane Database Syst Rev 2013;4.

9 Cohen MS, Chen YQ, McCauley M, et al. Prevention of HIV1 infection with early antiretroviral therapy. N Engl J Med 2011;365:493-505

10 Marcus JL, Glidden DV, McMahan V, et al. Daily oral emtricitabine/ tenofovir preexposure prophylaxis and herpes simplex virus type 2 among men who have sex with men. PLoS One 2014;9:e91513.

11 Celum C, Morrow RA, Donnell D, et al. Daily oral tenofovir and Emtricitabine-Tenofovir preexposure prophylaxis reduces herpes simplex virus type 2 acquisition among heterosexual HIV-1Uninfected men and women. Ann Intern Med 2014;161:11-19.

12 Shamseer L, Moher D, Clarke M, et al. Preferred reporting items for systematic review and meta-analysis protocols (PRISMA-P) 2015: elaboration and explanation. BMJ 2015;349:97647.

13 Moher D, Liberati A, Tetzlaff J, et al. Preferred reporting items for systematic reviews and meta-analyses: the PRISMA statement. PLoS Med 2009;6:e1000097.

14 Division of HIV/AIDS Prevention NCfHA, Viral Hepatitis, STD, and TB Prevention, Centers for Disease Control and Prevention. Preventing New HIV Infections. Hiv guidelines web site, 2018. Available: https:// www.cdc.gov/hiv/guidelines/preventing.html [Accessed $02 \mathrm{Apr}$ 2019].

15 FDA. Truvada fact sheet. US department of health and human services web site 2012, 2019.

16 Covidence systematic review software. Available: www.covidence. org

17 Higgins JPT, Thompson SG. Quantifying heterogeneity in a metaanalysis. Stat Med 2002;21:1539-58.

18 Deeks J, Dinnes J, D'Amico R, et al. Evaluating non-randomised intervention studies. Health Technol Assess 2003;7:1-173.

19 Higgins JPT, Altman DG, Gotzsche PC, et al. The Cochrane collaboration's tool for assessing risk of bias in randomised trials. BMJ 2011;343:d5928. 